\title{
Decidability and Undecidability of Marked PCP
}

\author{
Vesa Halava ${ }^{1}$, Mika Hirvensalo ${ }^{2,1 \star}$, and Ronald de Wolf 3,4 \\ 1 Turku Centre for Computer Science, Lemminkäisenkatu 14 A, 4th floor, FIN-20520, \\ Turku, Finland, vehalava@cs.utu.fi \\ 2 Department of Mathematics, University of Turku, FIN-20014, Turku, Finland. \\ mikhirve@utu.fi \\ 3 CWI, P.O. Box 94079, Amsterdam, The Netherlands, rdewolf@cwi.nl \\ 4 University of Amsterdam
}

\begin{abstract}
We show that the marked version of the Post Correspondence Problem, where the words on a list are required to differ in the first letter, is decidable. On the other hand, PCP remains undecidable if we only require the words to differ in the first two letters. Thus we locate the decidability/undecidability-boundary between marked and 2-marked PCP.
\end{abstract}

\section{Introduction: PCP and Marked PCP}

The Post Correspondence Problem (PCP) [6] is one of the most useful undecidable problems, because it can be simply described and many other problems can easily be reduced to it, particularly problems in formal language theory. The general form of the problem is as follows. An instance of PCP is a four-tuple $I=(\Sigma, \Delta, g, h)$, consisting of a finite source alphabet $\Sigma=\left\{a_{1}, \ldots, a_{n}\right\}$, a finite target alphabet $\Delta$ and two homomorphisms $g, h: \Sigma^{*} \rightarrow \Delta^{*}(g(a b)=g(a) g(b)$ and $h(a b)=h(a) h(b)$ whenever $\left.a, b \in \Sigma^{*}\right)$. It is enough to define $g, h: \Sigma \rightarrow \Delta^{*}$, the extension is just concatenation. PCP is the following decision problem:

Given $I=(\Sigma, \Delta, g, h)$, is there an $x \in \Sigma^{+}$such that $g(x)=h(x)$ ?

In other words, we have two lists of words $g\left(a_{1}\right), \ldots, g\left(a_{n}\right)$ and $h\left(a_{1}\right), \ldots, h\left(a_{n}\right)$ and we want to decide if there is a correspondence between them: are there $a_{i_{1}}, \ldots, a_{i_{k}} \in \Sigma$ such that $g\left(a_{i_{1}}\right) \ldots g\left(a_{i_{k}}\right)=h\left(a_{i_{1}}\right) \ldots h\left(a_{i_{k}}\right)$ ?

The general form of this problem is undecidable [6], the reason being that the two morphisms together can simulate the computation of a Turing machine on a specific input. Examining restricted versions of PCP allows one to determine the exact boundary between decidability and undecidability. For instance, the problem becomes trivially decidable (but NP-complete) if we ask for the existence of a solution $x$ of length at most some fixed $k$ [2, p. 228]. If we restrict to $g, h$ which have to be injective ( $g$ is injective if $x \neq y \Rightarrow g(x) \neq g(y)$ ), the problem remains undecidable [4]. Also $\operatorname{PCP}(7)$, where we restrict to $n=7$, is

\footnotetext{
* Supported by the Academy of Finland under grant 14047.
}

C. Meinel and S. Tison (Eds.): STACS'99, LNCS 1563, pp. 207-216, 1999

(C) Springer-Verlag Berlin Heidelberg 1999 
still undecidable [5], but $\mathrm{PCP}(2)$ is decidable [1]. As far as we know, decidability or undecidability is still open for $2<n<7$.

A further restriction which we will examine in this paper is to have $g$ and $h$ marked, which we formally define as follows. If $z$ is a string, we use $\operatorname{Pre} f_{k}(z)$ to denote the prefix of length $k$ of $z\left(\operatorname{Pre}_{k}(z)=z\right.$ if $\left.|z| \leq k\right)$. A homomorphism $g$ is $k$-marked if $g(a)$ and $g(b)$ are nonempty and have $\operatorname{Pref}_{k}(g(a)) \neq \operatorname{Pref}_{k}(g(b))$ whenever $a \neq b \in \Sigma$. An instance $I=(\Sigma, \Delta, g, h)$ of PCP is $k$-marked if both $g$ and $h$ are $k$-marked, and $k$-marked PCP is the PCP decision problem restricted to $k$-marked instances. We will abbreviate 1-marked to marked. If $I$ is marked then $g(a)$ and $g(b)$ start with a different letter whenever $a \neq b \in \Sigma$, which implies that $|\Sigma| \leq|\Delta|$. Without loss of generality we may assume $\Sigma \subseteq \Delta$. Markedness clearly implies injectivity: suppose $g$ is marked and $x \neq y \in \Sigma^{+}$, let $x=z a x^{\prime}$ and $y=z b y^{\prime}, a$ and $b$ being the first letter where $x$ and $y$ differ. Because of markedness we have $g(a) \neq g(b)$, hence $g(x)=g(z) g(a) g\left(x^{\prime}\right) \neq g(z) g(b) g\left(y^{\prime}\right)=g(y)$, so $g$ is injective. The converse does not hold. Consider for instance $\Sigma=\Delta=\{1,2\}$, $g(1)=11, g(2)=12$, then $g$ is injective but not marked.

The proof of decidability of $\mathrm{PCP}(2)$ in [1] is based on a reduction from arbitrary instances of $\mathrm{PCP}(2)$ to marked instances of generalized $\mathrm{PCP}(2)$. [1] then prove by means of extensive case analysis that marked generalized $\mathrm{PCP}(2)$ is decidable. In particular marked $\mathrm{PCP}(2)$ is decidable. Here we prove that marked PCP is decidable for any alphabet size. We will in fact show that marked PCP is in EXPTIME (the class of languages that can be recognized in time upper bounded by $2^{p(N)}$ for some polynomial $p$ of the input size $N$ ).

As stated above, PCP can be used for establishing the boundaries between decidability and undecidability. The main result of this paper is decidability of marked PCP. How much can we weaken the markedness condition before we lose decidability? We will show in Section 3 that 2-marked PCP is undecidable, thus locating the decidability/undecidability-boundary between 1-markedness and 2-markedness.

In another direction, we can weaken the markedness condition by only requiring $g$ and $h$ to be prefix morphisms ( $g$ is prefix if no $g\left(a_{i}\right)$ is a prefix or another $g\left(a_{j}\right)$ ) or even biprefix ( $g$ is biprefix if no $g\left(a_{i}\right)$ is a prefix or suffix of another $\left.g\left(a_{j}\right)\right)$. It turns out that biprefix PCP is undecidable [8]. ${ }^{1}$

\section{Marked PCP Is Decidable}

\subsection{A Simpler Decision Problem}

We would like to give a decision method for marked PCP. First we give an algorithm for the following simpler problem, which also occurs in [1, Section 6$]$ :

Given marked $I=(\Sigma, \Delta, g, h)$ and $a \in \Delta$, are there $x, y \in \Sigma^{+}$such that $g(x)=h(y)$ and $g(x)$ starts with $a ?$

\footnotetext{
${ }^{1}$ Clearly, a marked morphism is prefix. Both marked and biprefix PCP are special cases of injective PCP, but 2-marked PCP is not. See also at the end of Section 3.
} 
We do not look for $g(x)=h(x)$ here but only for $g(x)=h(y)$, and we additionally require that $g(x)$ starts with some specific $a \in \Delta$. For example, if $I$ has

$$
\begin{array}{llll}
g\left(a_{1}\right)=a_{1} & g\left(a_{2}\right)=a_{2} & g\left(a_{3}\right)=a_{3} a_{4} & g\left(a_{4}\right)=a_{4} \\
h\left(a_{1}\right)=a_{1} a_{3} & h\left(a_{2}\right)=a_{4} a_{2} & h\left(a_{3}\right)=a_{3} a_{3} & h\left(a_{4}\right)=a_{2} a_{2}
\end{array}
$$

then for $a=a_{1}$, a solution would be $x=a_{1} a_{3} a_{2}$ and $y=a_{1} a_{2}$.

The next algorithm decides the problem.

1. Set $G=H=\emptyset, i=j=1$.

2. If there are $x_{1}, y_{1} \in \Sigma$ such that $g\left(x_{1}\right)$ and $h\left(y_{1}\right)$ start with $a$, then set $x=x_{1}, y=y_{1}$

else goto 4 .

3. (a) If $g(x)=h(y)$, then print "solution $x=x_{1} \ldots x_{i}$ and $y=y_{1} \ldots y_{j}$ " and terminate.

(b) If $g(x)$ is not a prefix of $h(y)$ nor vice versa, then goto 4 .

(c) If $g(x) s=h(y)$, then do the following.

If $s \in G$ then goto 4 ; else set $i=i+1$ and $G=G \cup\{s\}$.

If there is an $x_{i}$ such that $g\left(x_{i}\right)$ and $s$ start with the same letter, then set $x=x x_{i}$ and goto 3 ; else goto 4 .

(d) If $g(x)=h(y) s$, then analogous to previous step.

4. Print "no solution" and terminate.

Informally, we are building $x=x_{1} \ldots x_{i}$ and $y=y_{1} \ldots y_{j}$, trying to achieve $g(x)=h(y)$. We add on a new $x_{i+1}$ as long as $g(x)$ is a proper prefix of $h(y)$ (i.e., $g(x) s=h(y)$ for some suffix $s$ ), and add on a new $y_{j+1}$ if $h(y)$ is a proper prefix of $g(x)$. Note that at each point such $x_{i+1}$ or $y_{j+1}$ are unique (if they exist) because of markedness; if they do not exist we know there is no solution. We keep track of the suffixes we have seen so far in the sets $G$ and $H$. Because the number of possible suffixes is finite, either the process terminates with a solution, or at some point a suffix is encountered for the second time, in which case we know the process will cycle forever and there is no solution.

The solutions produced by this algorithm are of minimal length. Note carefully that the whole procedure is deterministic, because $g$ and $h$ are marked. Furthermore, if $N$ is the length of the instance $I$ given as input (i.e., the number of bits needed to describe the instance), then this procedure runs in time polynomial in $N$. Namely, each $g\left(a_{i}\right)$ and $h\left(a_{i}\right)$ can have length at most $N$, and hence can have at most $N-1$ proper suffixes. Since there are only $2 n=O(N)$ different $g\left(a_{i}\right)$ and $h\left(a_{i}\right)$, there are only $O\left(N^{2}\right)$ different suffixes, hence the loop of the algorithm can be repeated at most $O\left(N^{2}\right)$ times. This loop itself takes $O(N)$ steps, because (1) to check if $g(x)=h(y)$ or $g(x) s=h(y)$ or $g(x)=h(y) s$, we only need to check the way $g(x)$ and $h(y)$ have been changed by the addition of the previous $x_{i}$ or $y_{j}$, and (2) searching for a new $x_{i}$ (in step c) or $y_{j}$ (in step d) can be done in $O(n)=O(N)$ steps. Therefore the whole procedure runs in $O\left(N^{3}\right)$ steps. 


\subsection{Reducing to Simpler Instances}

Consider an instance $I=(\Sigma, \Delta, g, h)$ of marked PCP: we have two marked homomorphisms $g, h: \Sigma^{+} \rightarrow \Delta^{+}$, where $\Sigma=\left\{a_{1}, \ldots, a_{n}\right\} \subseteq \Delta$, and we want to decide if there is an $x \in \Sigma^{+}$such that $g(x)=h(x)$. Below we describe an approach to decide $I$ by reducing it to an equivalent but simpler instance $I^{\prime}$ of marked PCP ("equivalent" meaning that $I$ has a solution iff $I^{\prime}$ has one).

Suppose $\Delta=\left\{a_{1}, \ldots, a_{l}\right\}, l \geq n$. We can run the procedure of the previous section for every $a_{i} \in \Delta$, yielding pairs of (minimal-length) solutions $\left(u_{1}, v_{1}\right), \ldots,\left(u_{l}, v_{l}\right)$ where $u_{i}, v_{i} \in \Sigma^{+}$and $g\left(u_{i}\right)=h\left(v_{i}\right)$ starts with $a_{i}$, or nonexistence of solutions for certain $i$. At most $n$ of the $a_{i}$ can have a solution. Without loss of generality assume $1, \ldots, m \leq n$ are the $i$ that have a solution. We can turn this into a new instance $I^{\prime}=\left(\Sigma^{\prime}, \Delta, g^{\prime}, h^{\prime}\right)$ of PCP, where $\Sigma^{\prime}=\left\{a_{1}, \ldots, a_{m}\right\}, g^{\prime}\left(a_{i}\right)=u_{i}$ and $h^{\prime}\left(a_{i}\right)=v_{i}$. Note that $g^{\prime}$ and $h^{\prime}$ are marked, so $I^{\prime}$ is an instance of marked PCP. Also, since the procedure of the previous section runs in $O\left(N^{3}\right)$ steps and has to be run $n$ times here, $I^{\prime}$ can be built from $I$ in $O\left(N^{4}\right)$ steps. The reduction from $I$ to $I^{\prime}$ preserves equivalence:

Lemma 1. If $I$ and $I^{\prime}$ are as above, then $I$ and $I^{\prime}$ are equivalent.

Proof. Note that every solution $x$ to $I$ must be built up from $u_{i}$ and $v_{i}$ : there must be $i_{1}, \ldots, i_{k}$ such that $x=u_{i_{1}} \ldots u_{i_{k}}=v_{i_{1}} \ldots v_{i_{k}}$. This is easy to see from the example in Figure 1. Here $u_{1}=a_{5} a_{3} a_{1}$ and $v_{1}=a_{5} a_{3}$ is a solution to the simpler problem for $a_{1}$, similarly $\left(a_{2} a_{4}, a_{1} a_{2}\right)$ is a solution for $a_{6}$ and $\left(a_{6} a_{3}, a_{4} a_{6} a_{3}\right)$ is a solution for $a_{2}$. Here $x=a_{5} a_{3} a_{1} a_{2} a_{4} a_{6} a_{3}$ is a solution to $I$, $x^{\prime}=a_{1} a_{6} a_{2}$ is a solution to $I^{\prime}$, related by $x=g^{\prime}\left(x^{\prime}\right)$.

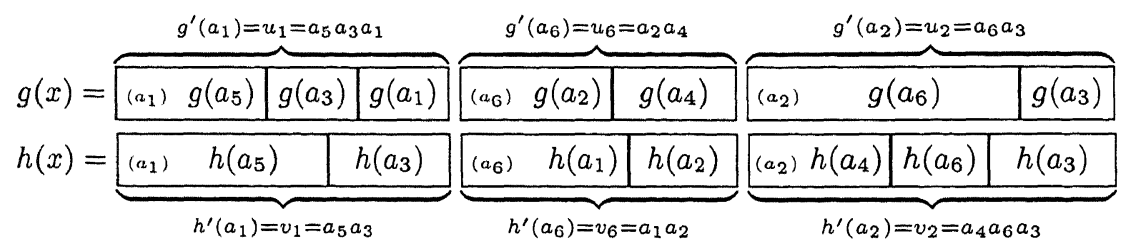

Fig. 1. How a solution to $I$ translates to $I^{\prime}$ and vice versa

In general, by construction, if $x^{\prime}$ is a solution to $I^{\prime}$ then $x=g^{\prime}\left(x^{\prime}\right)=h^{\prime}\left(x^{\prime}\right)$ is a solution to $I$. And conversely, for every solution $x$ to $I$ there is a solution $x^{\prime}$ o $I^{\prime}$ such that $x=g^{\prime}\left(x^{\prime}\right)=h^{\prime}\left(x^{\prime}\right)$. Thus $I$ and $I^{\prime}$ are equivalent.

If we could prove that $I^{\prime}$ is somehow simpler than $I$, then we could repeat the procedure, reduce to simpler and simpler equivalent instances $I^{\prime \prime}, I^{\prime \prime \prime}, \ldots$, and eventually decide $I$. There are at least two ways in which $I^{\prime}$ can be simpler than $I:\left|\Sigma^{\prime}\right|<|\Sigma|(m<n)$ or $\sigma\left(I^{\prime}\right)<\sigma(I)$, where $\sigma$ measures the "suffix complexity" of an instance $I=(\Sigma, \Delta, g, h)[1]$ :

$$
\begin{aligned}
\sigma(I) & =\mid \cup_{a \in \Sigma}\{x \mid x \text { is a proper suffix of } g(a)\} \mid \\
& +\mid \cup_{a \in \Sigma}\{x \mid x \text { is a proper suffix of } h(a)\} \mid
\end{aligned}
$$


If $n=m$, we would like $I^{\prime}$ to be simpler than $I$ in the sense that $\sigma\left(I^{\prime}\right)<\sigma(I)$. The following lemma shows that $I^{\prime}$ at least cannot be more complex than $I$ :

Lemma 2. If $I$ and $I^{\prime}$ are as above, then $\sigma\left(I^{\prime}\right) \leq \sigma(I)$.

Proof. Define the following four sets:

$$
\begin{aligned}
& G=\cup_{a \in \Sigma}\{x \mid x \text { is a proper suffix of } g(a)\} \\
& G^{\prime}=\cup_{a \in \Sigma^{\prime}}\left\{x \mid x \text { is a proper suffix of } g^{\prime}(a)\right\} \\
& H=\cup_{a \in \Sigma}\{x \mid x \text { is a proper suffix of } h(a)\} \\
& H^{\prime}=\cup_{a \in \Sigma^{\prime}}\left\{x \mid x \text { is a proper suffix of } h^{\prime}(a)\right\}
\end{aligned}
$$

We will define an injective function $p: G^{\prime} \rightarrow H$. Let $u \in G^{\prime}$, so $u$ is a proper suffix of some specific $g^{\prime}\left(a_{i}\right)=u_{i}=x_{1} \ldots x_{c}$ generated by the procedure of the previous section. Let $x_{r}$ be the first letter of $u$, and $s$ be the shortest suffix of some $h\left(y_{t}\right)$ due to which $x_{r}$ was added to $u_{i}$ in the procedure of the previous section, so $s$ is a prefix of $g\left(x_{r}\right)$ (see Figure 2) or vice versa. Define $p$ as $p(u)=s$.

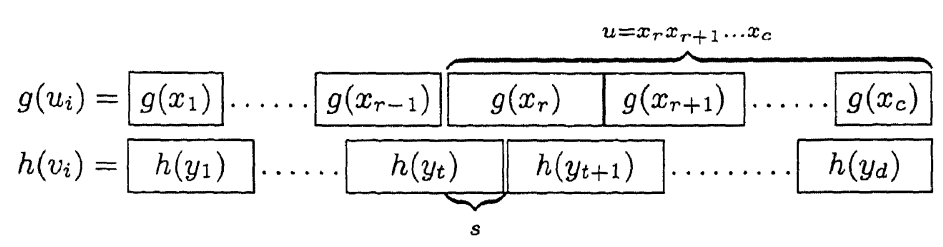

Fig. 2. The suffix $s$ corresponding to $u$

We will show $p$ is injective. If $u, u^{\prime} \in G^{\prime}$ and $p(u)=p\left(u^{\prime}\right)$, then $u$ and $u^{\prime}$ are associated with the same suffix $s=p(u)$, hence $u$ and $u^{\prime}$ must start with the same $x_{r}$ and (by determinism of the procedure of the previous section) continue in the same way, giving $u=u^{\prime}$. Thus $p$ is injective, which implies $\left|G^{\prime}\right| \leq|H|$.

Similarly we can define an injective function from $H^{\prime}$ to $G$, which proves $\left|H^{\prime}\right| \leq|G|$. It now follows that $\sigma\left(I^{\prime}\right)=\left|G^{\prime}\right|+\left|H^{\prime}\right| \leq|G|+|H|=\sigma(I)$.

\subsection{The Algorithm}

We will here give a method to decide if a given instance $I=(\Sigma, \Delta, g, h)$ of marked PCP has a solution. The idea is to make a sequence of equivalencepreserving reductions $I_{0}=I, I_{1}, I_{2}, \ldots$, such that once in a while a reduction from $I_{i}$ to $I_{i+1}$ simplifies the instance (makes the source alphabet or the suffix complexity smaller). We will show that either this sequence of reductions reaches an $I_{j}$ which has source alphabet of size 1 or $\sigma$ equal to 0 (so $I_{j}$ is decidable), or the sequence will repeat itself after a while and start cycling. Such cycles are detectable, and we will show that every $I$ leading to such a cycle is easily decidable.

So suppose the sequence of reductions does not reach an $I_{j}$ with alphabet of size 1 or $\sigma\left(I_{j}\right)=0$. Then it must get "stuck" at a certain source alphabet size 
and $\sigma$. That is, there exist a $k, m$ and $z$ such that all $I_{i}$ in the infinite sequence $I_{k}, I_{k+1}, I_{k+2}, \ldots$ have source alphabet of size $m$ and have $\sigma\left(I_{i}\right)=z$. Now this sequence must repeat itself after a while, for otherwise there would be infinitely many distinct instances with the same alphabet and $\sigma$-value, contradicting the next lemma.

Lemma 3. Let $\Sigma=\left\{a_{1}, \ldots, a_{m}\right\} \subseteq \Delta$ be finite sets and $z$ be a positive natural number. There exist only finitely many distinct instances $I=(\Sigma, \Delta, g, h)$ of $P C P$ that satisfy $\sigma(I) \leq z$.

Proof. An instance $I=(\Sigma, \Delta, g, h)$ is completely specified by giving the $2 m$ words $g\left(a_{1}\right), \ldots, g\left(a_{m}\right), h\left(a_{1}\right), \ldots, h\left(a_{m}\right) \in \Delta^{+}$. Note that if one of those words has length $>z+1$, then this word has more than $z$ proper suffixes and $\sigma(I)>z$. Accordingly, each of the $2 m$ words can have length at most $z+1$. There are $\sum_{i=1}^{z+1}|\Delta|^{i} \leq|\Delta|^{z+2}$ such words. Thus there are at most $|\Delta|^{(z+2) 2 m}$ choices for $2 m$ such words, and hence finitely many different $I$ that satisfy $\sigma(I) \leq z$.

This lemma shows that if the procedure does not converge to very simple instances then it will cycle, and we can detect this by noting that some $I_{k}$ and $I_{r}(k<r)$ are equal. It remains to show how we can decide such "cycling" instances of marked PCP. So suppose we have a cycle, assume without loss of generality that it already starts at $I_{0}$ :

$$
I_{0} \rightarrow I_{1} \rightarrow \cdots \rightarrow I_{r-1} \rightarrow I_{r}=I_{0},
$$

where $I_{i}=\left(\Sigma, \Delta, g_{i}, h_{i}\right)$. By the proof of Lemma 1 , for every solution $x_{i}$ to some $I_{i}$, there is a solution $x_{i+1}$ to $I_{i+1}$ such that $x_{i}=g_{i+1}\left(x_{i+1}\right)=h_{i+1}\left(x_{i+1}\right)$. Suppose $x_{0}$ is a solution to $I_{0}$ of minimal length. There must exist some solution $x_{r}$ to $I_{r}$ such that

$$
\begin{aligned}
& x_{0}=g_{1} g_{2} \ldots g_{r}\left(x_{r}\right) \\
& x_{0}=h_{1} h_{2} \ldots h_{r}\left(x_{r}\right)
\end{aligned}
$$

Since the $g_{i}$ and $h_{i}$ cannot be length-decreasing, we have $\left|x_{0}\right| \geq\left|x_{r}\right|$. But $x_{0}$ was chosen to be a minimal-length solution to $I_{0}$ and $x_{r}$ is also a solution to $I_{r}=I_{0}$, hence $\left|x_{0}\right|=\left|x_{r}\right|$. This implies that $g_{0}\left(=g_{r}\right)$ and $h_{0}\left(=h_{r}\right)$ map the letters occurring in $x_{r}$ to letters. But then the first letter of $x_{r}$ is already a solution, hence $\left|x_{0}\right|=\left|x_{r}\right|=1$. Thus $I_{0}$ has a solution iff $I_{0}$ has a 1-letter solution (i.e., there is an $a \in \Sigma_{0}$ such that $g_{0}(a)=h_{0}(a)$ ), and this is trivially decidable.

Below we summarize this analysis in an algorithm and a theorem:

\section{Decision procedure for marked PCP}

1. Set $\mathcal{I}=\emptyset, i=0, I_{0}=I$.

2. Set $i=i+1$.

3. Reduce $I_{i-1}$ to $I_{i}$ in the way stated above.

4. If $I_{i}$ has source alphabet of size 1 or $\sigma=0$, then decide $I_{i}$, print the outcome and terminate. 
5. If $I_{i}$ is simpler than $I_{i-1}$ (smaller source alphabet or $\sigma$ ) then set $\mathcal{I}=\emptyset$ and goto 2 .

6. If $I_{i} \in \mathcal{I}$ then there is a cycle and we can decide $I_{i}$ by checking if it has a 1-letter solution, print the outcome and terminate;

else set $\mathcal{I}=\mathcal{I} \cup\left\{I_{i}\right\}$ and goto 2 .

Theorem 1. Marked PCP is decidable.

\subsection{Complexity Analysis}

Let us analyze the complexity of this algorithm. Let $N$ be the length of the input instance $I$. Each reduction from $I_{i}$ to $I_{i+1}$ can be done in $O\left(N^{4}\right)$ steps. How many different reductions do we need to make? For a fixed alphabet size $|\Sigma| \leq$ $|\Delta|=m$ and suffix complexity $z$, we can make at most $m^{(z+2) 2 m}$ reductions before detecting a cycle (proof of Lemma 3). Since $m=O(N)$ and $z=O\left(N^{2}\right)$, this gives an upper bound of $2^{O\left(\log N \cdot N^{3}\right)}$ on the number of reductions for fixed alphabet size and suffix complexity. Alphabet size and suffix complexity cannot increase during the process. There are at most $n=O(N)$ different alphabet sizes and at most $\sigma(I)=O\left(N^{2}\right)$ different suffix complexities possible, so we have to make no more than $O\left(N^{3}\right) \cdot 2^{O\left(\log N \cdot N^{3}\right)}$ reductions. Since the set $\mathcal{I}$ can contain at most $2^{O\left(\log N \cdot N^{3}\right)}$ instances, the test $I_{i} \in \mathcal{I}$ in step 6 can be performed in $2^{O\left(\log N \cdot N^{3}\right)}$ steps. Thus the whole algorithm works in $2^{O\left(\log N \cdot N^{3}\right)}$ steps, which means that marked PCP is in EXPTIME.

\section{2-Marked PCP Is Undecidable}

Here we will show that if we weaken the condition of markedness, by only requiring the morphisms to be 2-marked, then PCP becomes undecidable again.

Consider the following semi-group $S_{7}$ with set of 5 generators $\Gamma=\{a, b, c, d, e\}$ and 7 relations:

$$
\begin{aligned}
& S_{7}=\langle a, b, c, d, e \mid R\rangle \\
& R=\{a c=c a, a d=d a, b c=c b, b d=d b, e c a=c e, e d b=d e, c c a=c c a e\}
\end{aligned}
$$

Tzeitin [10] (see also [7, p. 445]) proved that the following problem for this semi-group is undecidable:

Given $u, v \in \Gamma^{+}$, is $u=v \in S_{7}$ ?

Note that the set of 7 left-hand-sides of $R$ is 2-marked, and similarly for the set of 7 right-hand-sides of $R$. We will reduce this problem to 2-marked PCP. We use a slight modification of the standard reduction from word problems to $\mathrm{PCP}$, involving an alphabet with some underlined letters in order to ensure 2markedness.

Define the source alphabet as

$$
\Sigma=\Gamma \cup \underline{\Gamma} \cup\left\{B, E, \#, \underline{\#}, r_{1}, r_{2}, \ldots, r_{7}, \underline{r_{1}}, \underline{r_{2}}, \ldots, \underline{r_{7}}\right\},
$$


where $\underline{I}=\{\underline{a}, \underline{b}, \underline{c}, \underline{d}, \underline{e}\}$, and $r_{1}, \ldots, r_{7}$ are the 7 relations in $R$ and $\underline{r}_{1}, \ldots, \underline{r_{7}}$ are their underlined versions (considered as single letters), so $r_{1}=[\overrightarrow{a c}=c \bar{a}]$, $\underline{r_{1}}=[\underline{a c}=\underline{c a}]$ etc. Define the target alphabet as

$$
\Delta=\Gamma \cup \underline{\Gamma} \cup\{B, E, \#, \#\} .
$$

$B$ and $E$ will mark the beginning and end of expressions, respectively. Given $u, v \in \Gamma^{+}, g$ and $h$ are defined by Table 1 :

\begin{tabular}{|c||c|c|c|c|c|c|c|c|c|c|c|c|}
\hline & $B$ & $E$ & $\#$ & $\#$ & $a$ & $\ldots$ & $e$ & $\underline{a}$ & $\ldots$ & $\underline{e}$ & {$[s=t]$} & {$[\underline{s}=\underline{t}]$} \\
\hline \hline$g$ & $B u \#$ & $E$ & $\#$ & $\#$ & $\underline{a}$ & $\ldots$ & $\underline{e}$ & $a$ & $\ldots$ & $e$ & $\underline{t}$ & $s$ \\
\hline$h$ & $B$ & $\# v E$ & $\#$ & $\#$ & $a$ & $\ldots$ & $e$ & $\underline{a}$ & $\ldots$ & $\underline{e}$ & $s$ & $\underline{t}$ \\
\hline
\end{tabular}

Table 1. Definition of $g$ and $h$

Note that the constructed instance $I=(\Sigma, \Delta, g, h)$ is an instance of 2-marked $\mathrm{PCP}$. The following lemma shows that the reduction preserves equivalence with Tzeitin's problem:

Lemma 4. Let $u, v, I$ be as above. Then $u=v \in S_{7}$ iff $I$ has a solution.

Proof.

$\Longrightarrow$ : Suppose $u=v \in S_{7}$. Then there is a sequence $u=u_{1} \rightarrow u_{2} \rightarrow \cdots \rightarrow$ $u_{k}=v$, where $u_{i}=u^{\prime} s u^{\prime \prime}$ and $u_{i+1}=u^{\prime} t u^{\prime \prime}$, and $s=t \in R$ or $t=s \in R$. We construct a solution to $I$ by induction on $k$.

If $k=1$, then $u=v \in \Gamma^{+}$. Now $x=B u \# \underline{u} E$ is a solution to $I$.

Now let $I^{\prime}=\left(\Sigma, \Delta, g^{\prime}, h^{\prime}\right)$ be the instance of 2-marked PCP corresponding to $u=u_{k-1} \in S_{7}$. By the induction hypothesis we can assume that $I^{\prime}$ has a minimal-length solution $x^{\prime}$. It is easy to see that every solution must begin with $B$ and end with $E$, so $x^{\prime}=B y E$, and therefore $g^{\prime}(B y)=w \# u_{k-1}$ and $h^{\prime}(B y)=w$ for some $w$. Note that since $I$ and $I^{\prime}$ only differ in the assignment $h(E)$ and $h^{\prime}(E)$, and $E$ cannot occur in $y$ (because $x^{\prime}$ is minimal), we also have $g(B y)=w \# u_{k-1}$ and $h(B y)=w$. We distinguish two cases. Firstly, $u_{k-1}=$ $u^{\prime} s u^{\prime \prime}$ and $\bar{v}=u_{k}=u^{\prime} t u^{\prime \prime}$, where $r=[s=t]$ is one of the 7 relations. Then

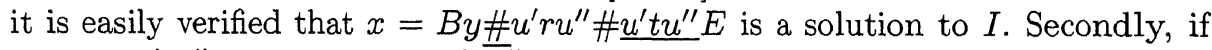
$u_{k-1}=u^{\prime} t u^{\prime \prime}$ and $v=u_{k}=u^{\prime} s u^{\prime \prime}$, then $x=B y \underline{\#} u^{\prime} t u^{\prime \prime} \# \underline{u^{\prime} r u^{\prime \prime} E}$ is a solution. This completes the induction step.

$\Longleftarrow$ : Suppose $I$ has a solution $x$. We can assume $x$ is of minimal length. This $x$ must be of the form $B x_{1} x_{2} \ldots x_{m} E$, where $x_{i} \in \Sigma$, so $g\left(B x_{1} \ldots x_{m} E\right)=$ $B u \# g\left(x_{1} \ldots x_{m}\right) E=h\left(B x_{1} \ldots x_{m} E\right)=B h\left(x_{1} \ldots x_{m}\right) \# v E$. Ignoring the underlining, $g(x)=h(x)$ must be of the form $B u_{1} \# u_{2} \# \ldots \# u_{k-1} \# u_{k} E$, where $u_{i} \in \Gamma^{*}, u_{1}=u$ and $u_{k}=v$. We will show that $u_{i}=u_{i+1} \in S_{7}$ for every $1 \leq i \leq k-1$, from which $u=v \in S_{7}$ follows.

Because \# occurs in $h\left(x_{1} \ldots x_{m}\right)$, there must be some least $i$ such that $x_{i}=$ $\#$, and hence $u=h\left(x_{1} \ldots x_{i-1}\right)$. Since there is no underlining in $u$, it follows that 
$x_{1}, \ldots, x_{i-1}$ must have been chosen from $a, \ldots, e, r_{1}, \ldots, r_{7}$. Let $x_{1} \ldots x_{i-1}=$ $w_{1} r_{i_{1}} w_{2} r_{i_{2}} \ldots w_{l}$, with $w_{i} \in \Gamma^{*}$ and $r_{i}=\left[s_{i}=t_{i}\right] \in\left\{r_{1}, \ldots, r_{7}\right\}$. Then $u=$ $h\left(w_{1} r_{i_{1}} w_{2} r_{i_{2}} \ldots w_{l}\right)=w_{1} s_{i_{1}} w_{2} s_{i_{2}} \ldots w_{l}$. See Figure 3 for illustration.

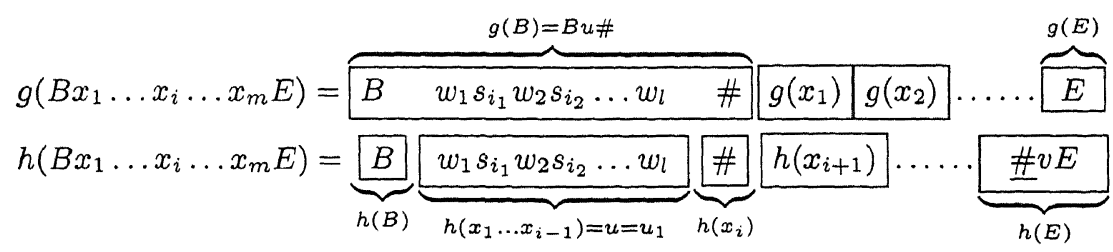

Fig. 3. Picture leading to $u=v$

Note that $g\left(x_{1} \ldots x_{i-1}\right)=g\left(w_{1} r_{i_{1}} w_{2} r_{i_{2}} \ldots w_{l}\right)=w_{1} t_{i_{1}} w_{2} t_{i_{2}} \ldots w_{l}$. But now, since we must have $g\left(x_{1} \ldots x_{m} E\right)=h\left(x_{i+1} \ldots x_{m} E\right)$, there must be a least $j>i$ such that $x_{j} \in\{\#, \#\}$ and $h\left(x_{i+1} \ldots x_{j-1}\right)=g\left(x_{1} \ldots x_{i-1}\right)=w_{1} t_{i_{1}} w_{2} t_{i_{2}} \ldots w_{l}$. The latter string (without underlining) is $u_{2}$. Note that $u_{1}=\overline{u_{2} \in S_{7} \text {, because }}$ $u_{1}(=u)$ and $u_{2}$ only differ by $u_{2}$ having $t_{i}$ where $u_{1}$ has $s_{i}$.

Continuing this reasoning, we can show that for every two words $u_{i}, u_{i+1} \in \Gamma^{*}$ occurring in $g(x)=h(x)$ separated by \#, ignoring underlining, we must have $u_{i}=u_{i+1} \in S_{7}$ (some of the words $u_{i}$ and $u_{i+1}$ may actually already be equal in $\left.\Sigma^{+}\right)$. Hence $u=v \in S_{7}$, since $g(x)$ starts with $u_{1}=u$ and ends with $u_{k}=v$.

Together with Tzeitin's result, the above lemma implies:

Theorem 2. 2-Marked PCP is undecidable.

To end this section, we note that 2-marked PCP is not a special case of injective PCP. For example, the morphism defined by $g(1)=23, g(2)=2, g(3)=$ 3 is 2 -marked but not injective. We can combine $k$-markedness and injectivity by calling a morphism $g$ strongly $k$-marked if $g$ is both $k$-marked and prefix (i.e., no $g\left(a_{i}\right)$ is a prefix of another $\left.g\left(a_{j}\right)\right)$. This clearly implies injectivity. It follows from a construction of Ruohonen [8] that strongly 5-marked PCP is undecidable: the biprefix instances of PCP constructed there to show undecidability of biprefix $\mathrm{PCP}$ are also 5 -marked. Decidability of strongly $k$-marked PCP for $1<k<5$ is still open.

\section{Conclusion and Future Work}

We can investigate the boundary between decidability and undecidability by examining which restrictions on the Post Correspondence Problem render the problem decidable. We have shown here that restricting PCP to marked morphisms gives us decidability. On the other hand, 2-marked PCP is still undecidable. 
The following questions are left open by this research:

- Is exponential time the best we can do when deciding marked PCP, or is there a polynomial-time algorithm for the problem?

- What about decidability of strongly $k$-marked PCP for $1<k<5$ ?

- What about decidability of marked generalized PCP $[1,3]$ ?

- The decidability status of PCP with elementary morphisms $[9, \mathrm{pp} .72-$ 77] is still open. A morphism $g$ is elementary if it cannot be written as a composition $g_{2} g_{1}$ via a smaller alphabet. Marked PCP is a subcase of elementary PCP which we have shown here to be decidable. Can our results help to settle the decidability status of elementary PCP?

\section{Acknowledgment}

We would like to thank Tero Harju, Juhani Karhumäki, and John Tromp for reading and commenting this paper, and Harry Buhrman for some discussions. The second author would like to thank the CWI for its hospitality during the summer of 1998 , when part of this work was done.

\section{References}

1. A. Ehrenfeucht, J. Karhumäki, and G. Rozenberg. The (generalized) Post correspondence problem with lists consisting of two words is decidable. Theoretical Computer Science, 21(2):119-144, 1982.

2. M. Garey and D. Johnson. Computers and Intractability: A Guide to the Theory of NP-Completeness. W. H. Freeman, 1979.

3. T. Harju, J. Karhumäki, and D. Krob. Remarks on generalized Post correspondence problem. In Proceedings of 13th STACS, volume 1046 of Lecture Notes in Computer Science, pages 39-48. Springer-Verlag, 1996.

4. Y. Lecerf. Récursive insolubilité de l'équation générale de diagonalisation de deux monomorphisms de monoïdes libres $\phi x=\Psi x$. Comptes Rendus Acad. Sci. Paris, 257:2940-2943, 1963.

5. Y. Matiyasevich and G. Sénizergues. Decision problems for semi-Thue systems with a few rules. In Proceedings of the 11th IEEE Symposium on Logic in Computer Science, pages 523-531, 1996.

6. E. L. Post. A variant of a recursively unsolvable problem. Bulletin of the American Mathematical Society, 52:264-268, 1946.

7. G. Rozenberg and A. Salomaa, editors. Handbook of Formal Languages, volume 1. Springer-Verlag, Berlin, 1997.

8. K. Ruohonen. Reversible machines and Post's correspondence problem for biprefix morphisms. Journal of Information Processing and Cybernetics (EIK), 21(12):579-595, 1985.

9. A. Salomaa. Jewels of Formal Language Theory. Pitman, 1981.

10. G. C. Tzeitin. Associative calculus with an unsolvable equivalence problem. Tr. Mat. Inst. Akad. Nauk, 52:172-189, 1958. In Russian. 